\title{
Overcoming Intrinsic Spin Resonance by Using an AC Dipole*
}

\author{
M. Bai ${ }^{1,2,3}$, L. Ahrens ${ }^{2}$, J.W. Glenn ${ }^{2}$, H. Huang ${ }^{2}$, S.Y. Lee ${ }^{1}$, L. Ratner ${ }^{2}$ \\ T. Roser ${ }^{2}$, M.J. Syphers ${ }^{2}$, W. van Asselt ${ }^{2}$, A. Yokosawa ${ }^{3}$ \\ ${ }^{1}$ Department of Physics, Indiana University, Bloomington, IN 47405, USA \\ ${ }^{2}$ Brookhaven National Laboratory, Upton, NY11973, USA \\ ${ }^{3}$ Argonne National Laboratory, 9700 Cass Ave., Argonne, Il 60493, USA
}

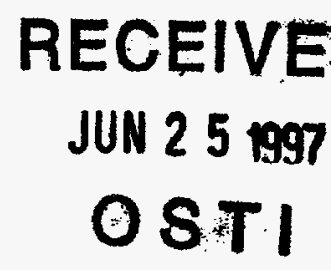

\begin{abstract}
Depolarization from an intrinsic spin resonance can be avoided by adiabatically exciting a coherent betatron oscillation. Experimental results of creating sustained coherent betatron oscillations in the Brookhaven National Laboratory AGS, and relevant spin tracking calculations are discussed.
\end{abstract}

\section{INTRODUCTION}

Intrinsic spin resonance is one of the major types of spin depolarizing resonances. It is caused by vertical betatron oscillations sampling the radial focusing field. The resonance condition is $G \gamma=K P \pm \nu_{z}$, where $K$ is an integer, $P$ is the number of superperiods, $\nu_{z}$ is the vertical betatron tune, $G=1.79$ is the anomalous g-factor for the proton, $\gamma$ is the Lorentz factor, and $G \gamma$ is the number of spin precession per orbit revolution.

For an isolated resonance with resonance strength $\epsilon$ at a constant acceleration rate $\alpha=\frac{d(G \gamma)}{d \theta}$ and $\theta$ as the bending angle, Froissart-Stora formula gives the ratio of the initial polarization $P_{i}$ before passing through this resonance to the final polarization $P_{f}$ after passing through it.

$$
\frac{P_{f}}{P_{i}}=2 e^{-\pi \frac{|\sigma|^{2}}{2 \alpha}}-1
$$

Based on Eq. 1, to avoid depolarization from spin resonances, coherent vertical betatron oscillation can be induced to increase the resonance strength of all particles to a level that an adiabatic spin flip will be induced with the normal acceleration rate of the synchrotron.

In this application, a controlled oscillation is adiabatically produced with a high frequency dipole magnet slowly energized to its final field amplitude and likewise slowly de-energized. Then the particle beam emittance is preserved.

This can easily be understood by studying the motion in a phase space frame which is rotating at the AC dipole oscillating frequency. Consider a particle influenced by a single horizontally oriented $\mathrm{AC}$ dipole field of length $\ell$ at a location in the accelerator where the vertical betatron amplitude function is $\beta_{z}$. The field oscillates according to $B_{x}=B_{m} \cos 2 \pi \nu_{m} n$, where $B_{m}$ is the amplitude

\footnotetext{
- The work performed under the auspices of the US Department of Energy
}

of the AC dipole field, $\nu_{m}$ is the modulation tune defined as the oscillating frequency divided by the accelerator's revolution frequency, and $n$ is the number of revolutions about the accelerator. In this frame, the net rotation an-

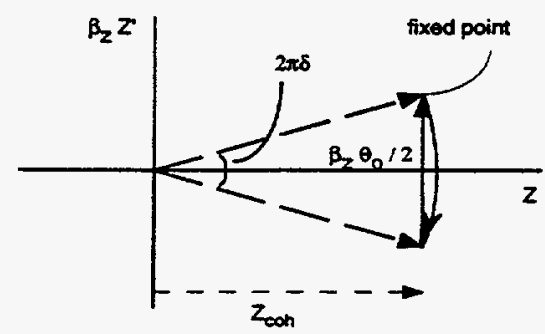

Figure 1: Phase space motion in the rotating frame at the maximum betatron amplitude location.

gle per turn of a particle's phase space vector is $2 \pi \delta$, with $\delta \equiv \nu_{z}-\left(k-\nu_{\mathrm{m}}\right)$ where $k$ is an integer and $\nu_{z}$ is the vertical betatron tune. The $\mathrm{AC}$ dipole field deflects the trajectory through an angle $\theta=B_{x} \ell /(B \rho)$. Since the dipole field is oscillating at the same frequency as the rotating frame, the time average change of $Z^{\prime}$ in this frame is just half the amplitude of the modulated angular deflection:

$$
\left\langle Z^{\prime}\right\rangle=\frac{1}{2}\left(B_{m} l\right) /(B \rho) \equiv \frac{1}{2} \theta_{0} .
$$

Therefore, the coherent betatron oscillation amplitude is given by

$$
2 \pi \delta \cdot Z_{c o h}=\left\langle Z^{\prime}\right\rangle \beta_{z} \rightarrow Z_{c o h}=\frac{B_{\mathrm{m}} \ell}{4 \pi(B \rho) \delta} \beta_{z} .
$$

Eq. 3 shows that the coherent betatron oscillation amplitude is proportional to the maximum dipole field strength and inversely proportional to the distance from the resonant tune. This equation can also be obtained by working out the Hamiltonian[1].

\section{EXPERIMENTAL TESTS IN THE AGS}

To investigate the feasibility of this procedure, tests were performed in the Brookhaven AGS with unpolarized beam during a recent Heavy Ion Physics run. The AC dipole was located where the vertical betatron function was about $16.6 \mathrm{~m}[2]$. 


\subsection{Experimental Set-up}

For the beam tests, the AC dipole was linearly ramped up in about $3 \mathrm{~ms}$ and kept at full amplitude for about $4 \mathrm{~ms}$ then ramped back to zero in another $3 \mathrm{~ms}$. During acceleration in the AGS, the revolution frequency changes as the particle momentum increases. The AGS beam RF signal was used as the external clock for an arbitrary function generator to generate the sine wave with fixed tune. Thus, by multiplying this sine wave with the magnet's strength amplitude envelope, an amplitude modulated sine wave with a fixed tune was obtained.

The experiment was performed in the AGS with $\mathrm{Au}^{+77}$ beam. Its normalized emittance (95\%) was about $5 \pi \mathrm{mm}$ $\mathrm{mrad}$ and the rms beam size at the working energy ( $1.1 \mathrm{GeV} / \mathrm{c}$ per nucleon) is about $3.9 \mathrm{~mm}$ at a maximum amplitude function location.

Transverse beam position measurements[2] were obtained with a pick-up electrode (PUE) located 4.5 betatron oscillations downstream of the AC dipole, where the vertical amplitude function is $15.4 \mathrm{~m}$. The beam transverse profile was measured using the exsiting AGS IPM (Ionization Profile Monitor) system[2].

\subsection{Results}

The coherence amplitude as a function of distance between the $\mathrm{AC}$ dipole modulation tune from the intrinsic betatron tune was measured both during acceleration as well as at fixed energy in the AGS. The AC dipole modulation tune function was held fixed and the betatron tune was changed to produce different tune separations.

A typical example of the readback from the $\mathrm{AC}$ dipole current transformer as a function of revolution number is shown in the bottom portion of Fig. 2 . Here, the full dipole strength is $2.82 \mathrm{G}-\mathrm{m}$ and its modulation tune is 0.75 .
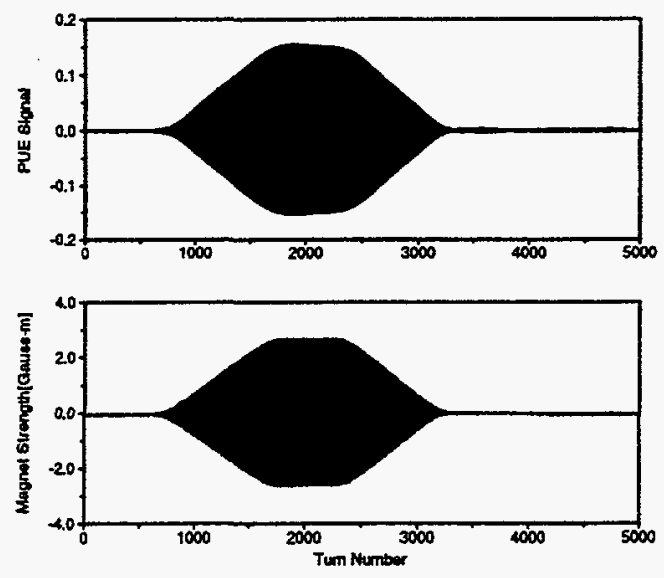

Figure 2: Transverse displacement (top) and AC dipole magnet field amplitude (bottom) as a function of revolutions about the AGS.

The top part of Fig. 2 is the beam's average vertical displacement sampled turn-by-turn. It shows that the coherent betatron motion nicely follows the AC dipole field ampli- tude shown in the bottom portion of the figure. The intrinsic betatron tune is 8.745 .

Results from a typical emittance scan is shown in Fig. 3. The top portion of the figure shows the beam size (rms,
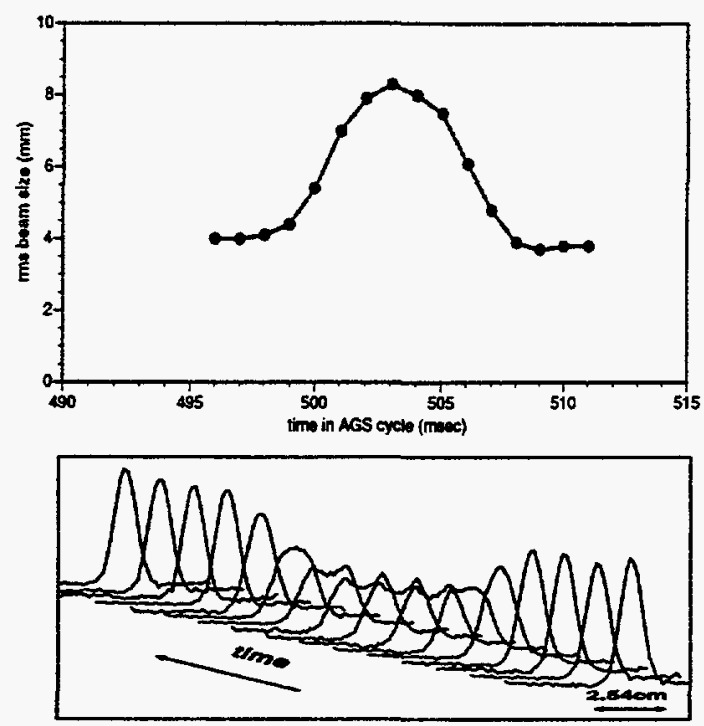

Figure 3: Measured transverse rms beam size versus time in AGS cycle (top). Corresponding beam profiles versus time in AGS cycle (bottom).

in $\mathrm{mm}$ ) versus time, with measurements taken in $1 \mathrm{~ms}$ intervals. The bottom part of the figure shows a mountain range plot of the corresponding vertical beam profiles. The broadening of the distribution and narrowing as the $\mathrm{AC}$ magnet turns off is evident.

The measurement time of the IPM system was $3 \mathrm{~ms}$, and hence the beam circles the AGS roughly 900 times during the measurement. Thus, the rms beam size shown in the plot is the time averaged value. The maximum "measured" rms beam size is related to the actual rms beam size, $\sigma_{0}$, and the oscillation amplitude $Z_{\text {con }}$ generated by the AC dipole by

$$
\sigma_{\text {meas }}=\sigma_{\circ} \sqrt{1+\frac{1}{2}\left(\frac{Z_{\text {coh }}}{\sigma_{0}}\right)^{2}} .
$$

The fact that the measured rms beam size returns to its previous value indicates that the process was indeed adiabatic and that the beam emittance was preserved.

The ratio of the measured coherent amplitude to dipole field amplitude is shown in Fig. 4 as a function of different tune separations between the modulation tune and the betatron tune. During this set of measurements, the momentum was held fixed at $1.1 \mathrm{GeV} / \mathrm{c}$ per nucleon. This particular ratio is shown because when the tunes were placed near resonance without reducing the dipole field amplitude, the oscillation amplitude would become large enough to induce beam loss. Thus, only smaller amplitude oscillations provided meaningful measurements at these tune separations. 
For a tune separation of 0.01 , the largest amplitude oscillation which could be maintained without significant beam loss was 2.6 times the rms beam size.

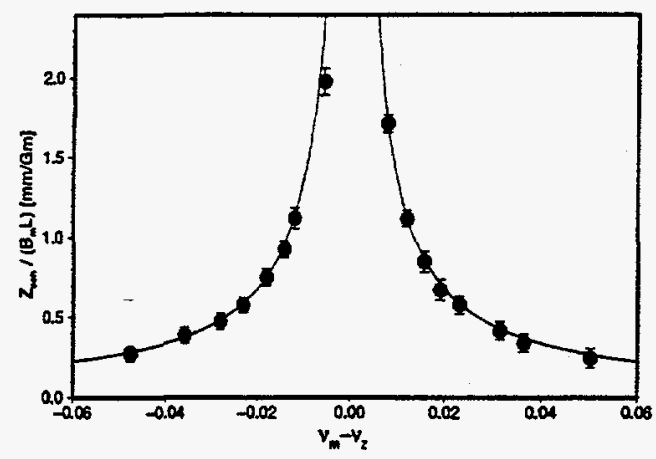

Figure 4: Normalized betatron oscillation amplitude versus $\delta$. The solid line is the predicted curve using Eq. 3 .

A similar set of measurements is depicted in Fig. 5, where in this case the beam is being accelerated at a rate

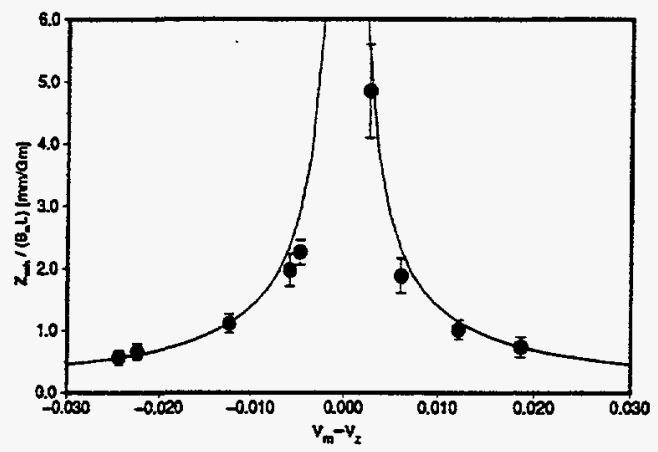

Figure 5: Normalized betatron oscillation amplitude versus $\delta$. The solid line is the predicted curve using Eq. 3 .

of $\dot{\gamma} \equiv \frac{d \gamma}{d t}=2.44 \mathrm{sec}^{-1}$ The arbitrary function generator was used to generate the appropriate sine wave with the AGS beam RF signal used as the clock signal. Fig. 5 shows that by accurately fixing the tune separation, the general features of the AC dipole induced coherent betatron oscillations are the same for acceleration and storage.

\section{SPIN TRACKING}

Multiple particle spin tracking was done with AGS polarized proton parameters. Fig. 6 shows the simulation of crossing $G \gamma=0+\nu_{x}$, where the vertical betatron tune $\nu_{z}=8.65$ and the horizontal betatron tune $\nu_{x}=8.85$. The normalized $95 \%$ transverse emittance $\epsilon_{x}=\epsilon_{y}=15 \pi \mathrm{mm}$ mrad. The acceleration rate $\alpha=4.5 \times 10^{-5}$. The modulation tune $\nu_{m}$ is 0.01 away from $\nu_{x}$. The spin is fully flipped at $G \gamma=8,9$ by the $5 \%$ partial snake. The spin flip at the $G \gamma=0+\nu_{2}$ is enhanced from $59 \%$ to $95 \%$ by the $\mathrm{AC}$ dipole running at the field strength $20 \mathrm{G}-\mathrm{m}$. Further improvement can be achieved by obtaining smaller beam emittance or closer distance between the modulation tune $\nu_{m}$ and the vertical betatron tune $\nu_{z}$.

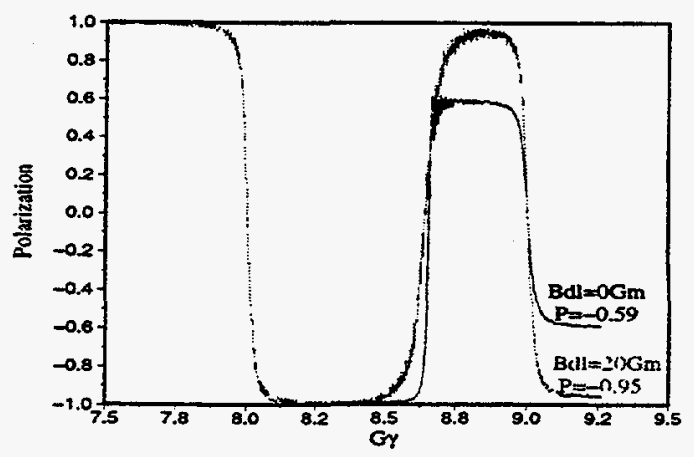

Figure 6: Spin tracking results

\section{CONCLUSION}

Sustained coherent transverse beam oscillations have been achieved in the AGS using a high frequency $A C$ driven dipole magnet. The amplitude of the oscillations using the present system has been as large as 2.6 times the rms beam size, and the oscillations were held at this level for over 1000 revolutions. By adiabatically increasing and decreasing the dipole field amplitude during this procedure, the transverse emittance of the beam was left unaffected. To set the scale, had an oscillation of that amplitude been left to oscillate freely, the beam emittance would have increased by a factor of 7 after filamentation. This result provides encouragement that this technique can be used to induce spin flip of polarized proton beams in synchrotrons when crossing strong intrinsic depolarizing resonances during acceleration. Another application of this device in a polarized beams storage ring would be the reversal of the polarization direction of the beam. By slowly sweeping the frequency of the modulated dipole field through the spin precession frequency of the storage ring, an adiabatic spin flip can be induced, thus reversing the polarization direction of each individual particle in the storage ring without increasing the beam emittance. This can be useful for reducing systematic errors in polarized beam experiments.

\subsection{Acknowledgments}

The authors would like to thank A. Dunbar, D. Gassner, J. Reich, P. Sampson, R. Sanders, G. Smith, C. Whalen, N. Williams, $\mathrm{K}$. Zeno for their help during the experiment.

\section{REFERENCES}

[1] M. Bai, S. Y. Lee, H. Huang, T. Roser, M. Syphers, "Overcoming the Intrinsic Spin Resonance using Resonance Island created by RF Dipole," AGS/RHIC/SN No. 055

[2] M. Bai et al,"Adiabatic Excitation of Coherent Betatron Oscillation with an AC Dipole field", to be submitted to Phys. Rev. E. 


\section{DISCLAIMER}

This report was prepared as an account of work sponsored by an agency of the United States Government. Neither the United States Government nor any agency thereof, nor any of their empleyees, makes any warranty, express or implied, or assumes any legal liability or responsibility for the accuracy, completeness, or usefulness of any information, apparatus, product, or process disclosed, or represents that its use would not infringe privately owned rights. Reference herein to any specific commercial product, process, or service by trade name, trademark, manufacturer, or otherwise does not necessarily constitute or imply its endorsement, recommendation, or favoring by the United States Government or any agency thereof. The views and opinions of authors expressed herein do not necessarily state or reflect those of the United States Government or any agency thereof. 


\section{DISCLAIMER}

Portions of this document may be illegible in electronic image products. Images are produced from the best available original document. 